Published in final edited form as:

J Psychosom Res. 2014 January ; 76(1): 19-22. doi:10.1016/j.jpsychores.2013.11.011.

\title{
Binge eating and menstrual dysfunction
}

\author{
Monica Ålgars, Ph.D. ${ }^{1}$, Lu Huang, M.S. ${ }^{2}$, Ann F. Von Holle, M.S. ${ }^{2}$, Christine M. Peat, Ph.D. ${ }^{2}$, \\ Laura Thornton, Ph.D. ${ }^{2}$, Paul Lichtenstein, Ph.D. ${ }^{3}$, and Cynthia M. Bulik, Ph.D. ${ }^{2,4}$
}

${ }^{1}$ Department of Psychology and Logopedics, Abo Akademi University, Turku, Finland ${ }^{2}$ Department of Psychiatry, University of North Carolina at Chapel Hill ${ }^{3}$ Department of Medical Epidemiology and Biostatistics, Karolinska Institutet, Stockholm, Sweden ${ }^{4}$ Department of Nutrition, University of North Carolina at Chapel Hill

\section{Abstract \\ Objective-The relation between eating disorders and menstrual function has been widely studied, but it is unknown whether the behavior of binge eating itself is related to menstrual dysfunction.}

Methods-The 11,503 women included in this study were from the Swedish Twin study of Adults: Genes and Environment. The associations between menstrual dysfunction and binge eating were analyzed using logistic regression or multiple linear regression models with generalized estimation equations.

Results-Women who reported lifetime binge eating were more likely to report either amenorrhea or oligomenorrhea than women who reported no binge eating. These results persisted when controlling for compensatory behaviors including self-induced vomiting, laxative use, and diuretic use. No differences between women with and without a history of binge eating were observed for age at menarche.

Conclusion-Even when controlling for the effect of compensatory behaviors, the behavior of binge eating is associated with menstrual dysfunction. Metabolic and endocrinological factors could underlie this association. Careful evaluation of menstrual status is warranted for women with all eating disorders, not just anorexia nervosa.

\section{Keywords}

menstruation; amenorrhea; oligomenorrhea; binge eating; binge eating disorder

(C) 2013 Elsevier Inc. All rights reserved.

Correspondence to: Dr. Bulik, Department of Psychiatry, University of North Carolina at Chapel Hill, CB \#7160, Chapel Hill, NC 27599-7160, Voice: (919) 8431689 Fax: (919) 843-8802, cbulik@ med.unc.edu.

Publisher's Disclaimer: This is a PDF file of an unedited manuscript that has been accepted for publication. As a service to our customers we are providing this early version of the manuscript. The manuscript will undergo copyediting, typesetting, and review of the resulting proof before it is published in its final citable form. Please note that during the production process errors may be discovered which could affect the content, and all legal disclaimers that apply to the journal pertain.

Conflicts of Interest: Other authors report no biomedical financial interests or potential conflicts of interest.

Competing Interest Statement

All authors have completed the Unified Competing Interest form at http://www.icmje.org/coi_disclosure.pdf and declare that the authors have no competing interests to report. 


\section{INTRODUCTION}

Menstrual function can be disrupted in both adolescent and adult women who suffer from eating disorders $(1,2)$. Although recently eliminated as a diagnostic criterion for anorexia nervosa (AN) (3), amenorrhea, defined as the absence of three consecutive menstrual periods, has been a central feature of that disorder historically and may be an index of severity (4). In addition, oligomenorrhea, or irregular menstruation, occurs in about half of girls and women with bulimia nervosa $(\mathrm{BN})(1,2,5)$. Obesity is also associated with menstrual irregularities (6-8). Yet, despite these documented associations, to our knowledge, no previous study has specifically examined the association between binge eating and menstrual dysfunction.

Factors contributing to the complex relationship between eating psychopathology and menstrual dysfunction include nutritional status and metabolic disturbances, which can interfere with the complex interplay of gonadotropin and gonadal hormones that are essential for reproductive function (6). In AN, amenorrhea is thought to be related to a gonadotropin deficiency caused by malnutrition and extreme weight-regulatory behaviors (9) and, likewise, menstrual irregularities in BN have been hypothesized to be secondary to disruption of the hypothalamic-pituitary-gonadal axis due to restricting energy intake and purging $(1,2)$. Although binge eating is a central feature of $\mathrm{BN}$ and may also occur in $\mathrm{AN}$, the extent to which the symptom of binge eating alone might contribute to menstrual irregularity is unknown. Binge eating is of particular interest as it affects approximately 5\% of adult women (10), is a primary diagnostic criterion for both $\mathrm{BN}$ and binge eating disorder (BED), and is also strongly associated with obesity $(11,12)$.

$\mathrm{BN}$ is also associated with polycystic ovary syndrome (PCOS), which is marked by menstrual irregularities/disruption $(13,14)$ secondary to insulin resistance-mediated testosterone increases (6). Higher levels of testosterone are associated with anovulation and menstrual irregularities $(15,16)$ and insulin is a regulator of testosterone levels $(17)$. In a similar vein, the effects of obesity on reproductive function are primarily mediated through hormonal changes (5). Decreased concentrations of sex hormone binding globulin in women with central adiposity lead to higher levels of free testosterone, which, in turn, inhibits follicular maturation, resulting in anovulation (6).

It has been suggested that binge eating may be a contributing factor in the expression of both PCOS and menstrual irregularities because gross fluctuations in energy intake affect insulinresistance (18). In a study of women seeking fertility treatment, BED was more common among infertile women than fertile controls (19). Also, nearly $25 \%$ of women with PCOS meet criteria for BED (20).

In the present study, we investigated the association between lifetime binge eating and menstrual dysfunction in a large sample of female twins. We hypothesized that binge eating would be positively associated with menstrual dysfunction. In addition, we conducted exploratory analyses on a smaller subsample to examine whether individuals meeting criteria for BED would be significantly more likely to report menstrual dysfunction than individuals in the referent group.

\section{METHOD}

Participants

Participants were from the population-based, prospective Swedish Twin study of Adults: Genes and Environment (STAGE), which is a cohort of the Swedish Twin Registry born between 1959 and 1985 (STR; http://ki.se/ki/jsp/polopoly.jsp?d=9610\&l=en) (21, 22). Data 
for STAGE were collected in 2005 when participants were 20-47 years old using web-based questionnaires with a computer-assisted telephone interview option. Over 25,000 individuals responded (response rate $=59.6 \% ; 14,000+$ women). A total of $13,295(94.2 \%)$ women provided information for the eating disorders section. Women who were missing information regarding age at interview, highest lifetime weight and height, lifetime binge eating (absent or present), BED criteria, menstrual history, or PCOS diagnosis were excluded from the present study $(n=1792 ; 13.5 \%)$. The resulting sample comprised 11,503 women ( $81.5 \%$ of all women who responded to STAGE).

STAGE was approved by both the Regional Ethics Committee at the Karolinska Institutet and by the Biomedical Institutional Review Board at the University of North Carolina at Chapel Hill. For more extensive descriptions of STAGE, see Furberg (21) and Lichtenstein (22).

\section{Measures}

BMI and Weight Categories-Lifetime highest BMI $\left(\mathrm{kg} / \mathrm{m}^{2}\right)$ was calculated from height (meters) and weight $(\mathrm{kg})$ information that participants provided regarding the time when they were at their heaviest. To examine whether binge eating would be associated with menstrual dysfunction in women across different weight categories, lifetime highest BMI was categorized as underweight $<18.50$; normal weight $=18.50-24.99$; overweight $=$ $25.00-29.99$; or obese $\geq 30.00 \mathrm{~kg} / \mathrm{m}^{2}$.

Binge Eating and Binge Eating Disorder-Binge eating was assessed with two questions: "Have you ever had eating binges when you ate what most people would regard as an unusually large amount of food in a short period of time?" and "When you were having eating binges, did you feel that your eating was out of control?" Binge eating was scored as present if respondents endorsed both eating an unusually large amount of food and any loss of control. BED was defined by DSM-IV-TR criteria (23) and considered present if the participant: 1) endorsed binge eating as defined above (Criterion A);2) endorsed at least three of the following symptoms: eating faster than usual, eating until uncomfortably full, eating large amounts of food when not hungry, eating alone due to embarrassment, and feeling disgusted/depressed/guilty after overeating (Criterion B); 3) indicated that binge eating made them feel somewhat, very much, or extremely upset or distressed (Criterion C); 4) reported a minimum of four binge eating episodes in a one month period (modified, broadened Criterion D); and 5) did not engage in inappropriate compensatory behaviors (e.g., laxative use, diuretic use, diet pills, fasting/not eating, excessive exercising, and vomiting) during the time when they were binge eating (Criterion $\mathrm{E})$. In addition, conservatively, a lifetime history of AN or BN was a rule-out for BED $(n=9)$.

In the medical checklist section of the STAGE questionnaire, participants were asked a general question about having or ever having had "anorexia/bulimia/eating disorders." Response options were "yes" or "no." The agreement between the response on the medical checklist and eating disorder diagnosis assigned using the diagnostic algorithms was $\kappa=$ 0.40 . This lower value could reflect the lack of specificity of the checklist question. Unlike the specific diagnostic criteria questions, the checklist item could be open to a number of interpretations (e.g., an individual who never had a formal diagnosis could have answered no whereas the diagnostic algorithm would have scored her positive for the diagnosis). Of greater relevance, of those women who had a history of binge eating as defined above, $66.2 \%$ responded "yes" to the general question about ever having "anorexia/bulimia/eating disorders." 
Compensatory Behaviors-Compensatory behaviors were defined as engaging in laxative use, diuretic use, or vomiting during the same period of time that binge eating was occurring, or having used laxatives, diuretics or vomiting at least once or twice in their lifetime to control weight or shape. Thus, compensatory behaviors were scored as present if participants reported engaging in laxative use, diuretic use, or vomiting in the context of a binge or independent of a binge episode as a method of weight or shape control.

Menstrual Dysfunction-Menstrual dysfunction was evaluated using two variables. Response options were "yes" and "no" except where indicated. Amenorrhea was considered present if participants endorsed "Have you ever missed more than three periods without any natural causes such as pregnancy or menopause?" Lifetime oligomenorrhea was considered present if participants endorsed "Have you, during any period of your life, had irregular menstrual periods defined as more than five weeks between periods?" For PCOS, participants were asked whether they had ever been diagnosed with PCOS. For age at menarche, each participant was asked to report how old she was when her menstrual cycles started.

\section{Statistical Analyses}

All analyses were conducted with SAS software (version 9.2) from SAS Institute Inc., (2008). Two different types of models estimated the association between both binge eating and BED as independent variables and menstrual function as outcome variables. Separate logistic regression models were used to estimate the odds of amenorrhea or lifetime oligomenorrhea in the groups reporting binge eating or BED compared with the respective groups reporting no history of binge eating or BED. Mean differences in age at menarche between binge eating groups were estimated with linear regression models. For all models, BMI category was entered as a covariate along with the interaction between binge eating or BED with BMI category. The interaction was not significant for any model; thus it was removed and models including binge eating and BMI categories were reapplied. PCOS and a dichotomous variable indicating the presence or absence of compensatory behaviors were included as covariates for all menstrual measures, and age was included as a covariate for models evaluating the outcomes amenorrhea and lifetime oligomenorrhea.

Generalized estimation equations (GEE) were used to account for the nonindependence of the data due to inclusion of twin pairs. All p-values were corrected by false discovery rate (24) to control for type-I error. One result of this approach is the possibility of obtaining confidence intervals that do not include unity, but are not statistically significant.

\section{RESULTS}

\section{Sample Description}

In our final sample, 676 (5.9\%) women endorsed lifetime binge eating and of those 59 $(0.5 \%)$ participants met criteria for BED. The mean age at menarche (range $7-25$ years) and prevalence of menstrual dysfunction in women who reported binge eating and in the subset of women with BED are presented in Table 1 . The number of women who endorsed binge eating in each weight class was: $1(0.8 \%)$ in the underweight category, $333(4.8 \%)$ in the normal weight category, $203(6.4 \%)$ in the overweight category, and $139(10.7 \%)$ in the obese category. The number of women who met criteria for BED in each weight class was: 0 $(0.0 \%)$ in the underweight category, $22(0.3 \%)$ in the normal weight category, $17(0.5 \%)$ in the overweight category, and $20(1.5 \%)$ in the obese category. 


\section{Associations Between Binge Eating and Menstrual Dysfunction}

Results of models assessing the association between binge eating and menstrual dysfunction are presented in Table 2. Binge eating was significantly associated with amenorrhea ( $\mathrm{p}<$. 001) and lifetime oligomenorrhea ( $\mathrm{p}<.001)$ after controlling for PCOS, compensatory behaviors, and age. No significant differences between women with and without a history of binge eating were observed for age at menarche.

\section{Associations Between Binge Eating Disorder and Menstrual Dysfunction}

The results from logistic regression and multiple regression analyses evaluating associations between menstrual dysfunction and BED are presented in Table 3. None of the associations between BED and measures of menstrual function were significant.

\section{DISCUSSION}

In the present study we examined the associations between lifetime binge eating and menstrual dysfunction in a large population-based sample of Swedish female twins. We also conducted exploratory analyses evaluating the associations between BED and menstrual function in a smaller subsample. The prevalence of lifetime binge eating and BED were $5.9 \%$ and $0.5 \%$, respectively, which is comparable to that of other European countries but lower than the prevalence observed in American nonclinical samples $(10,25-27)$. The prevalence of lifetime amenorrhea was $10.4 \%$ and the prevalence of lifetime oligomenorrhea was $33.7 \%$. Previous studies have reported prevalence estimates between $2.6 \%$ and $7.6 \%$ for amenorrhea and $11.3 \%$ for oligomenorrhea; however, these studies included younger women or reported one-year point prevalences $(28,29)$. Controlling for regular compensatory behaviors, PCOS, BMI, and age, women who reported lifetime binge eating were significantly more likely to report amenorrhea and oligomenorrhea than women who did not report lifetime binge eating. Although the correlational nature of our data does not allow conclusions about causality to be drawn, some testable hypotheses can be forwarded. It is possible that the behavior of binge eating itself may affect menstrual function independent of weight because binge eating may interfere with hormonal function that, in turn, affects menstrual function. The interplay between eating pathology and menstrual dysfunction appears to be mediated by disruption of insulin and gonadal hormone function (6). Eating binges tend to be high in fat and carbohydrates (30). When eating foods rich in carbohydrates, blood glucose levels increase and insulin is released in response (31). Thus, recurrent binge eating can increase insulin levels, which, via resultant increases in testosterone, could influence follicular maturation and ovulation, resulting in menstrual dysfunction. Another potential explanation for the detected association is that underlying hormonal dysregulation could lead to both binge eating (14) and menstrual dysfunction (32). It is also possible that the irregular hormonal fluctuations that drive the menstrual irregularities exacerbate binge eating. Also, emotional eating varies across the menstrual cycle (33). Irregular menstruation could lead to irregular appetite cycles that, due to their unpredictability, could be distressing and increase the risk of binge eating.

No significant associations between BED and any measure of menstrual function were found. However, a numerically greater proportion of women with BED than women without BED reported amenorrhea or oligomennorhea. Further studies are needed to replicate and confirm this finding. No significant differences between women with and without binge eating were found for age at menarche. Previous research findings on this topic have been mixed; in some studies binge eating has been associated with early menarche, while others have failed to detect an association $(34,35)$. 
The results should be interpreted in the context of the study's limitations. All information about eating behaviors and menstrual dysfunction was based on computer-based self-reports, which may affect reliability (36). On the other hand, participants may be more likely to give accurate reports on sensitive matters in an anonymous computer-based study (37). BMI was calculated based on the participants' self-reported weight when they were at their heaviest with no measured heights or weights available for validation. The variables used to evaluate amenorrhea and oligomenorrhea only included "yes" and "no" response options, which could complicate comparisons with studies including more comprehensive measures of menstrual dysfunction. The response rate was $\sim 60 \%$ and information on whether nonparticipants differed from participants on the study variables was not available. Response bias can therefore not be ruled out. Finally, the sample consisted of Swedish women, which may limit generalizability to other populations with different observed prevalences of binge eating and $\operatorname{BED}(25,27)$. Future research should further investigate the association between binge eating and menstrual dysfunction and the metabolic or hormonal disruption that may underlie this association in larger, more ethnically diverse samples. In addition, the present study did not include data regarding the onset of binge eating or menstrual dysfunction. Longitudinal data are needed to understand the temporal relationship between binge eating and menstrual dysfunction

In sum, the present study is, to our knowledge, the first to investigate the association between binge eating and menstrual dysfunction. The results revealed significant associations between binge eating and both amenorrhea and oligomenorrhea, even when controlling for compensatory behaviors. Our results suggest that menstrual dysfunction is not limited to anorexia nervosa and underscore the importance clinically of thoroughly assessing menstrual status in women with all forms of eating psychopathology.

\section{Acknowledgments}

Source of Funding: This study was supported by grants CA-085739 (P.I.: P.F. Sullivan) and AI-056014 (P.I.: P.F. Sullivan) from the National Institutes of Health. The Swedish Twin Registry is supported by grants from the Swedish Department of Higher Education and the Swedish Research Council. Dr. Peat is supported by the National Institute of Mental Health grant T32 MH076694 (PI: Bulik). Dr. Bulik is a consultant for Shire Pharmaceuticals.

\section{Glossary}

$\begin{array}{ll}\text { AN } & \text { anorexia nervosa } \\ \text { BN } & \text { bulimia nervosa } \\ \text { BED } & \text { binge eating disorder } \\ \text { PCOS } & \text { polycystic ovary syndrome } \\ \text { BMI } & \text { body mass index }\end{array}$

\section{REFERENCES}

1. Vyver E, Steinegger C, Katzman DK. Eating disorders and menstrual dysfunction in adolescents. Ann N Y Acad Sci. 2008; 1135:253-264. [PubMed: 18574232]

2. Poyastro Pinheiro A, Thornton LM, Plotonicov KH, Tozzi F, Klump KL, Berrettini WH, et al. Patterns of menstrual disturbance in eating disorders. Int J Eat Disord. 2007; 40(5):424-434. [PubMed: 17497704]

3. American Psychiatric Association. Diagnostic and statistical manual of mental disorders. 5th ed. 2013.

4. Hebebrand J, Bulik CM. Critical appraisal of the provisional DSM-5 criteria for anorexia nervosa and an alternative proposal. Int J Eat Disord. 2011; 44(8):665-678. [PubMed: 22072403] 
5. Seidenfeld ME, Rickert VI. Impact of anorexia, bulimia and obesity on the gynecologic health of adolescents. Am Fam Physician. 2001; 64(3):445-450. [PubMed: 11515833]

6. Zain MM, Norman RJ. Impact of obesity on female fertility and fertility treatment. Womens Health (Lond Engl). 2008; 4(2):183-194. [PubMed: 19072520]

7. Norman RJ, Clark AM. Obesity and reproductive disorders: a review. Reprod Fertil Dev. 1998; 10(1):55-63. [PubMed: 9727593]

8. Lake JK, Power C, Cole TJ. Women's reproductive health: the role of body mass index in early and adult life. Int J Obes Relat Metab Disord. 1997; 21(6):432-438. [PubMed: 9192225]

9. Winston AP. The clinical biochemistry of anorexia nervosa. Ann Clin Biochem. 2012; 49(Pt 2): 132-143. [PubMed: 22349551]

10. Abebe DS, Lien L, Torgersen L, von Soest T. Binge eating, purging and non-purging compensatory behaviours decrease from adolescence to adulthood: A population-based, longitudinal study. BMC Public Health. 2012; 12(32)

11. Stunkard AJ. Eating disorders and obesity. Psychiatr Clin North Am. 2011; 34(4):765-771. [PubMed: 22098802]

12. de Zwaan M. Binge eating disorder and obesity. Int J Obes Relat Metab Disord. 2001; 25:51-55.

13. Raphael FJ, Rodin DA, Peattie A, Bano G, Kent A, Nussey SS, et al. Ovarian morphology and insulin sensitivity in women with bulimia nervosa. Clin Endocrinol (Oxf). 1995; 43(4):451-455. [PubMed: 7586620]

14. Morgan JF, McCluskey SE, Brunton JN, Hubert Lacey J. Polycystic ovarian morphology and bulimia nervosa: a 9-year follow-up study. Fertil Steril. 2002; 77(5):928-931. [PubMed: 12009345]

15. Haning RV Jr, Hackett RJ, Flood CA, Loughlin JS, Zhao QY, Longcope C. Testosterone, a follicular regulator: key to anovulation. J Clin Endocrinol Metab. 1993; 77(3):710-715. [PubMed: 8370694]

16. Van Anders SM, Watson NV. Menstrual cycle irregularities are associated with testosterone levels in healthy premenopausal women. AM J Hum Biol. 2006; 18(6):841-844. [PubMed: 17039468]

17. Nestler JE. Insulin regulation of human ovarian androgens. Hum Reprod. 1997; 12:53-62. [PubMed: 9403321]

18. McCluskey SE, Lacey JH, Pearce JM. Binge-eating and polycystic ovaries. Lancet. 1992; 340(8821):723. [PubMed: 1355813]

19. Sbaragli C, Morgante G, Goracci A, Hofkens T, De Leo V, Castrogiovanni P. Infertility and psychiatric morbidity. Fertil Steril. 2008; 90(6):2107-2111. [PubMed: 18462733]

20. Kerchner A, Lester W, Stuart SP, Dokras A. Risk of depression and other mental health disorders in women with polycystic ovary syndrome: a longitudinal study. Fertil Steril. 2009; 91(1):207212. [PubMed: 18249398]

21. Furberg H, Lichtenstein P, Pedersen NL, Thornton L, Bulik CM, Lerman C, et al. The STAGE cohort: a prospective study of tobacco use among Swedish twins. Nicotine Tob Res. 2008; 10(12): 1727-1735. [PubMed: 18988069]

22. Lichtenstein P, Sullivan PF, Cnattingius S, Gatz M, Johansson S, Carlstrom E, et al. The Swedish Twin Registry in the third millennium: an update. Twin Res Hum Genet. 2006; 9(6):875-882. [PubMed: 17254424]

23. American Psychiatric Association. Diagnostic and Statistical Manual of Mental Disorders. Fourth Edition Text Revision. Washington, DC: American Psychiatric Press; 2000.

24. Benjamini Y, Hochberg Y. Controlling the false discovery rate: A practical and powerful approach to multiple testing. Journal of the Royal Statistical Society Series B (Methodological). 1995; 57(1):289-300.

25. Hudson JI, Hiripi E, Pope HG Jr, Kessle RC. The prevalence and correlates of eating disorders in the National Comorbidity Survey Replication. Biol Psychiatry. 2007; 61(3):348-358. [PubMed: 16815322]

26. Kessler RC, Berglund PA, Chiu WT, Deitz AC, Hudson JI, Shahly V, et al. The prevalence and correlates of binge eating disorder in the World Health Organization World Mental Health Surveys. Biol Psychiatry. 2013; 73(9):904-914. [PubMed: 23290497] 
27. Grucza RA, Przybeck TR, Cloninger CR. Prevalence and correlates of binge eating disorder in a community sample. Compr Psychiatry. 2007; 48(2):124-131. [PubMed: 17292702]

28. Bachmann GA, Kemmann E. Prevalence of oligomenorrhea and amenorrhea in a college population. Am J Obstet Gynecol. 1982; 144(1):98-102. [PubMed: 7114117]

29. Münster K, Helm P, Schmidt L. Secondary amenorrhoea: prevalence and medical contact- -a crosssectional study from a Danish county. Br J Obstet Gynaecol. 1992; 99(5):430-433. [PubMed: 1622918]

30. Bartholome LT, Raymond NC, Lee SS, Peterson CB, Warren CS. Detailed analysis of binges in obese women with binge eating disorder: Comparisons using multiple methods of data collection. Int J Eat Disord. 2006; 39(8):685-693. [PubMed: 16937383]

31. Coulston AM, Liu GC, Reaven GM. Plasma glucose, insulin and lipid responses to highcarbohydrate low-fat diets in normal humans. Metabolism. 1983; 32(1):52-56. [PubMed: 6336816]

32. Neinstein LS. Menstrual dysfunction in pathophysiologic states. West J Med. 1985; 143(4):476484. [PubMed: 3911586]

33. Klump KL, Keel PK, Racine SE, Burt SA, Neale M, Sisk CL, et al. The interactive effects of estrogen and progesterone on changes in emotional eating across the menstrual cycle. $\mathrm{J}$ Abnorm Psychol. 2013; 122(1):131-137. [PubMed: 22889242]

34. Reichborn-Kjennerud T, Bulik CM, Sullivan PF, Tambs K, Harris JR. Psychiatric and medical symptoms in binge eating in the absence of compensatory behaviors. Obes Res. 2004; 12(9):14451454. [PubMed: 15483209]

35. Stice E, Presnell K, Bearman SK. Relation of early menarche to depression, eating disorders, substance abuse, and comorbid psychopathology among adolescent girls. Dev Psychol. 2001; 37(5):608-619. [PubMed: 11552757]

36. Crockett LJ, Schulenberg JE, Petersen AC. Congruence between objective and self-reported data in a sample of young adolescents. J Adolesc Res. 1987; 2(4):383-392.

37. Bangsberg DR, Bronstone A, Hofmann R. A computer-based assessment detects regimen misunderstandings and nonadherence for patients on HIV antiretroviral therapy. AIDS care. 2002; 14(1):3-15. [PubMed: 11798401] 


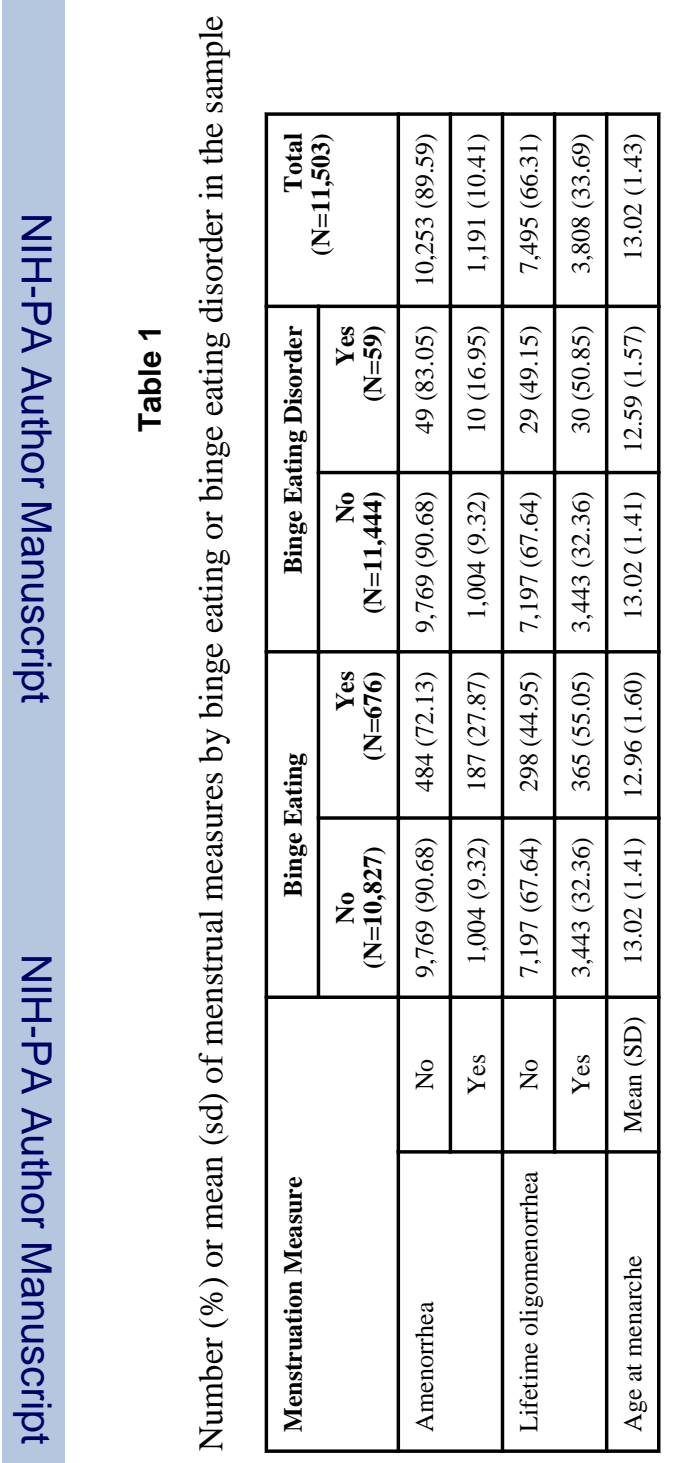


Table 2

Adjusted odds ratios or mean difference in women (with $95 \%$ confidence intervals indicated in parentheses) who had binge-eating behavior compared with those who did not, controlling for PCOS, BMI, age, and compensatory behaviors.

\begin{tabular}{|l|r|r|}
\hline Menstruation Measure & Estimate (95\% CI) & $\chi^{\mathbf{2}}$ (FDR-p) \\
\hline Amenorrhea & $\mathrm{OR}=2.60(2.07,3.26)$ & $50.35(<.001)$ \\
\hline Lifetime oligomenorrhea & $\mathrm{OR}=1.79(1.49,2.14)$ & $36.60(<. \mathbf{0 0 1})$ \\
\hline Age at menarche & Mean difference $=0.05(-0.09,0.18)$ & $0.50(.516)$ \\
\hline
\end{tabular}

Note. FDR $=$ false discovery rate. 


\section{Table 3}

Adjusted odds ratio or mean difference (indicated in parentheses) of having each menstruation measure in women who endorse binge eating disorder versus those who do not.

\begin{tabular}{|l|r|r|}
\hline Menstruation Measure & Estimate (95\% CI) & $\chi^{\mathbf{2}}$ (FDR-p) \\
\hline Amenorrhea & $\mathrm{OR}=1.62(0.76,3.43)$ & $2.73(.196)$ \\
\hline Lifetime oligomenorrhea & $\mathrm{OR}=1.88(1.08,3.27)$ & $1.19(.432)$ \\
\hline Age at menarche & Mean difference $=-0.32(-0.69,0.05)$ & $0.12(.731)$ \\
\hline
\end{tabular}

$\mathrm{FDR}=$ false discovery rate. 